\title{
On The Computation of Secondary Electron Emission Models
}

\author{
Sébastien Clerc, John R. Dennison, Ryan Hoffmann, Johnathan Abbott
}

\begin{abstract}
Secondary electron emission is a critical contributor to the charge particle current balance in spacecraft charging. Spacecraft charging simulation codes use a parameterized expression for the secondary electron yield $\delta\left(\mathrm{E}_{0}\right)$ as a function of incident electron energy Eo. Simple three-step physics models of the electron penetration, transport and emission from a solid are typically expressed in terms of the incident electron penetration depth at normal incidence $R\left(E_{0}\right)$, and the mean free path of the secondary electron, $\lambda$. We recall classical models for the range $R\left(E_{0}\right)$ : a power law expression of the form $b_{1} E_{0}{ }^{n 1}$, and a more general empirical double power law $R\left(E_{0}\right)=b_{1} E_{0}{ }^{n 1}+b_{2} E_{0}{ }^{n 2}$. In most models, the yield is the result of an integral along the path length of incident electrons. An improved fourth-order numerical method to compute this integral is presented and compared to the standard second-order method. A critical step in accurately characterizing a particular spacecraft material is the determination of the model parameters in terms of the measured electron yield data. The fitting procedures and range models are applied to several measured data sets to compare their effectiveness in modeling the function $\delta\left(E_{0}\right)$ over the full range of energy of incident particles.
\end{abstract}

Index Terms-Electron emission, Secondary Electron Yield, Surface charging

\section{INTRODUCTION}

S econdary electron emission (SEE) is often the largest contributor in the charged particle current balance driving spacecraft charging in space plasma. Most spacecraft surfaces are generally covered with low yield materials (metals, graphite), which leads to large negative absolute potential during charging events. On the other hand, some dielectric materials such as glass have a high secondary emission yield and build up large positive differential potentials. This can result in an inverse gradient situation, a major source of electrostatic discharges. Another important aspect of secondary emission is its strong variation with the incident energy, which leads to threshold effects (see [1]). As noted in [2], an accurate modeling of SEE is therefore crucial to the simulation of spacecraft charging. It is particularly critical to get the correct behavior at high incident energy, since the flux of electrons in the plasmasheet during charging events is

Manuscript received December 1, 2005. S. Clerc is with Alcatel Alenia Space, Research Department, Cannes La Bocca, 06156 France, (phone / fax: +33 492926052 / 970, e-mail: Sebastien.Clerc@AlcatelAleniaSpace.com)

J. R. Dennison, R. Hoffmann and J. Abbott are with the Physics Department, Utah State University, , Logan, UT 84322-4415 USA, (phone: 435-797-2936; fax: 435-7972492; e-mail: JR.Dennison@usu.edu). peaked at some tens of $\mathrm{keV}$.

Standard convention distinguishes between secondary electrons as those with energy below $50 \mathrm{eV}$, and backscattered electrons as those with higher energies, up to the incident energy. The back-scattering current is generally smaller than the true secondary emission current by one order of magnitude in typical space environments. For this reason, we will concentrate on true SEE in this paper.

Measuring SEE properties of materials is a particularly difficult task [3]. It is especially true for dielectric materials, because the implanted charges and the resulting internal electric field modify the trajectories of the incident and secondary electrons [4], [5]. This point is however outside the scope of the present study.

Modeling SEE is also a difficult and important point. Spacecraft charging simulation codes require that measured SEE yield curves be fit to parametric models. Although all the models of the literature correctly predict a SEE maximum around $100-700 \mathrm{eV}$, they differ greatly in their predictions of the asymptotic dependence at high incident energy. A fundamental source of difference is the way the incident electron penetration and energy dissipation inside the material is described. The models may also differ in the way they account for the propagation of secondary electrons (SE).

After a brief review of SEE models, we concentrate on numerical approximation issues and show their influence on the computation of the SEE yield curve and parameter-fitting procedures. We will propose some simple fitting strategies and apply them to different types of materials.

\section{THREE-STEP MODELS FOR SEE}

\section{A. Introduction}

In standard theories SEE is modeled as a three-step process: first is the production of secondary electrons (SEs) at a depth $z$ by incident penetrating electrons, followed by the transport of these SE towards the surface; and finally the emission of SE across the surface barrier.

\section{B. SE production}

The production of SE is the result of the energy loss of incident particles. The number of created secondary electrons per unit volume per second can be expressed as

$$
d N / d t=-\operatorname{div}(j E) / I,
$$

where $j E$ is the energy flux and $I$ is the effective energy 
necessary to create one SE. Next, we suppose that the current density of incident particles $j$ is constant up to the range of incident particles. The local rate of SE production is therefore proportional to the incident current density (or number flux) $j$,

$$
\frac{d N}{d t}=-j \frac{1}{I}\left(\frac{d E}{d z}\right) \text {. }
$$

At this point, a model for the energy deposition profile $d E(z) / d z$ for a given incident energy $E_{0}$ is needed. This is where theoretical models differ most. We detail these differences in section III.

\section{Transport and emission}

Only a fraction of the SEs will actually reach the surface and be emitted, while the remaining part will either recombine with holes, get trapped or contribute to the conduction. It is generally sufficient to use a single model of SE transport and emission for the whole SE population, regardless of the modulus or direction of their velocity.

Let $\tau(z)$ be the probability for a given SE created at a depth $z$ to reach the surface and be emitted. Summing the contribution of SE created at any depth, one gets the following expression for the SEE yield:

$$
\delta=-\int_{0}^{R\left(E_{0}\right)} \frac{d E}{d z} \tau(z) d z / I \text {. Error! }
$$

\section{Bookmark not defined.}

The escape potential $\tau(z)$ has to be a decreasing function of the depth vanishing at infinity. The most common choice is a decreasing exponential

$$
\tau(z)=C \exp (-z / \lambda)
$$

where $\lambda$ is related to the SE mean free path [9, 12-14]. Another possible choice for $\tau(z)$ is a rational fraction [6].

Alternatively, Jonker (see e.g. [7,8]) assumes that the probability density function of SE decreases with the path length instead of the depth. When the emission depth $Z$ increases, only the electrons with a velocity directed toward the surface will be able to reach it. The Jonker escape potential decreases therefore faster than an exponential. This potential involves an additional integration over velocity angles which must be computed numerically.

A more complete model could also include energy effects, particularly the influence of the potential barrier which SEs must overcome to be emitted.

\section{ENERGY DEPOSITION PROFILE}

\section{A. The Continuous Slowing Down Approximation}

In the Continuous Slowing Down Approximation (CSDA), the effect of inelastic collisions on the incident particle is modeled as a continuous braking force which depends only on the current value of the energy. In other words, there is no history effect. In paragraph II.B, we have assumed that the current of incident particles is constant up to the range, and then drops to zero: this is also a consequence of the CSDA.

If the variation of the range with the incident energy $R(E)$ is known, then the stopping power $S$ can be expressed from its derivative as $S(E)=1 / R^{\prime}(E)$, and the energy is related to the depth through $z=R\left(E_{0}\right)-R(E)$.

\section{B. The CSDA stopping power and the Bragg curve}

For all types of particles and scatterers, the curve of $S(E)$ presents a maximum, decreases and reaches a plateau at high energy when radiation becomes the principal mode of energy dissipation. The stopping power maximum corresponds to a maximum efficiency of energy transfer between the incident particle and the scatterers. It occurs at lower energy for low density materials and low mass particles. On Fig. 1, we depict the stopping power profile for three metals, as given in the NIST interactive database [9]. For materials used on spacecraft, the stopping power maximum occurs at energies lower than $1 \mathrm{keV}$. Experiments as well as Monte Carlo simulations are difficult to perform at such a low energy level, but we can assume that such a peak exists nevertheless.

However the CSDA range is usually approximated by a monotonic power law $R(E)=b E^{n}$ [10], most often with $n \approx$

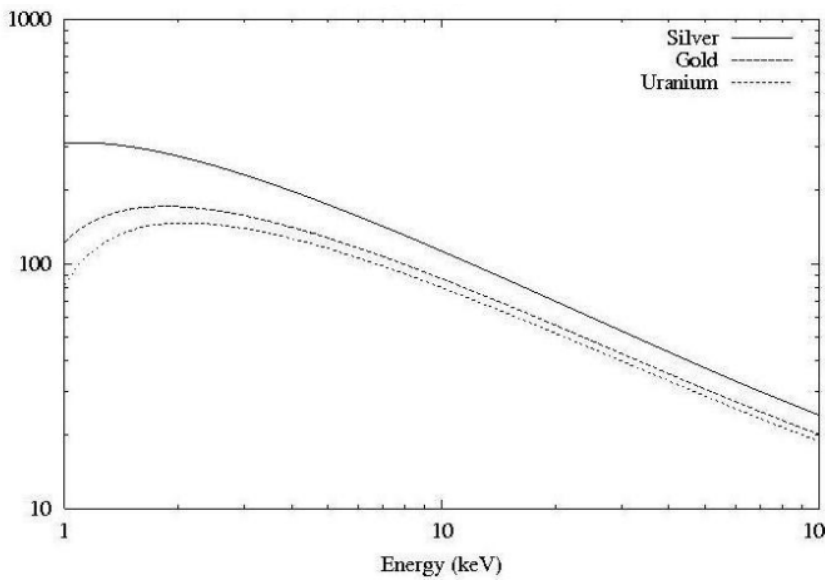

Figure 1. Continuous Slowing Down Approximation (CSDA). Stopping power (in $\mathrm{eV} \mathrm{m}^{3} / \mathrm{kg}$ ) versus incident energy for some metals, in log-log scale. NIST model [9].

1.35 (see e.g. [6], [11], [12], [13]). With $n>1$, the stopping power is infinite at zero incident energy and decreases continuously. The upper limit on $n$ is 2 [14].

On the other hand, Mandell et al. [15] propose an empirical double power law for the range which can be adjusted to fit the value of the stopping power maximum. Taking

$$
R(E)=b_{1} E^{n_{1}}+b_{2} E^{n_{2}}
$$

with $n_{1}<1$ and $n_{2}>1$, we get

$$
S(E)=E /\left[b_{1} n_{1} E^{n_{1}}+b_{2} n_{2} E^{n_{2}}\right]
$$

The stopping power reaches its maximum at the following energy

$$
E_{\text {Bragg }}=\left[-\frac{b_{1} n_{1}\left(n_{1}-1\right)}{b_{2} n_{2}\left(n_{2}-1\right)}\right]^{1 /\left(n_{2}-n_{1}\right)}
$$

From the expression for $S(E)$, one can derive the CSDA 


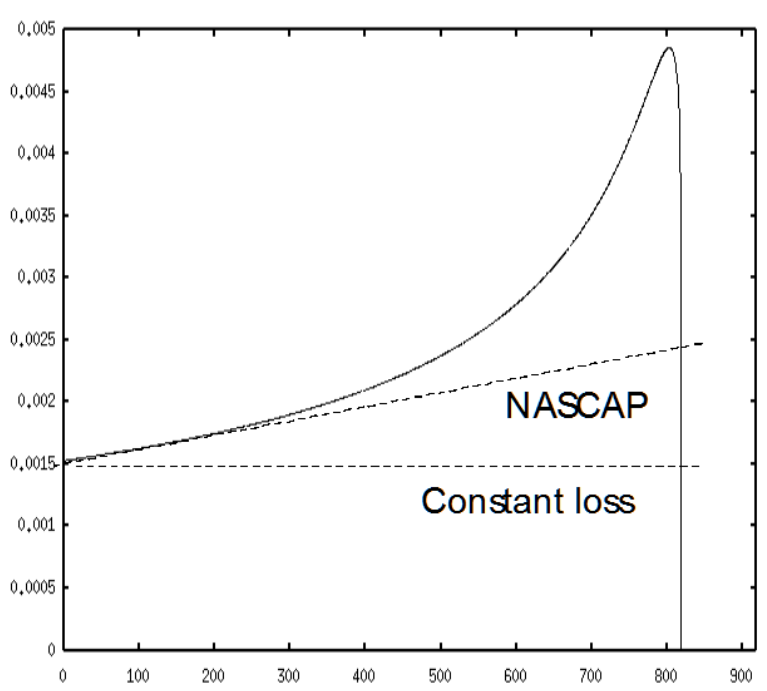

Figure 2: The Bragg curve (Stopping power versus depth). Dashed lines: zeroth order approximation or constant loss model (CLM) and linear approximation (NASCAP).

energy deposition profile $-d E / d z=S(E(z)$ ) (see Fig. 2.) This curve is known as the Bragg curve, and it is characterized by a strong peak near the maximum penetration depth of the trajectory, when the incident particle reaches the energy of the stopping power maximum, $E=E_{\text {Bragg. }}$

\section{Other effects}

Several effects tend to modify the energy deposition profile. They are all the more important for light incident particles such as electrons, and result in the smoothing of the Bragg peak.

The first of these is straggling, which is due to the statistical dispersion of collisions. The CSDA range is the average value of the range, but the stopping power $1 / R^{\prime}(E)$ deduced from this law is not exactly the average value of the energy deposition. The statistical effect is obviously more important for trajectories with multiple collisions, i.e. at higher incident energy.

A second and probably more important shortcoming of the CSDA comes from the angular scattering of incident particles. In fact, Monte Carlo simulations show that the average volume where the energy deposition of a particle occurs resembles an ellipsoid, whereas the CSDA assumes that everything occurs along a straight line. Because angular scattering is less important at high velocity, the ellipsoid is more elongated at high incident energy.

Finally, the SEs themselves can create free electrons in a cascading process, further spreading the affected volume of the incident particle [12].

Whereas the CSDA presents a situation where most of the energy is deposited near the end of the trajectory, these additional effects tend to spread the energy deposition more evenly inside an affected volume of a more complicated shape. Another consequence is the fact that the energy deposition profile at higher incident energy is no longer a translation of the profile at lower incident energy because of memory effects in the trajectory.

\section{Models of affected volume shape}

Schwarz [12] and Cazaux [13] both consider an affected volume of ellipsoidal shape where the energy of the incident particle is deposed evenly. After a two-dimensional integration, the energy deposition profile becomes

$$
(d E / d z) \approx E_{0} \sigma(z) / V,
$$

where $\sigma$ is the section of the affected volume at depth $z$, and $V$ is the total affected volume. Assuming an ellipsoidal shape for the affected volume yields a quadratic function for $\sigma$. The integral (3) in the definition of $\delta$ can therefore be computed analytically.

The Schwarz and Cazaux models differ in the geometry of the ellipsoid for a given material and incident energy. If $z_{c}$ is the distance of the center of the ellipsoid (most probable energy dissipation depth) and $z_{\max }$ the position of the deepest tip of the ellipsoid, then the ratio $k=z_{c} / z_{\max }$ is a materialdependent constant in Cazaux' model, whereas it increases with the incident energy in Schwarz' model. A less significant difference lies in the power used for the range law: 1.35 for Cazaux, 4/3 for Schwarz.

\section{E. Conclusion}

The CSDA and the affected volume approaches concentrate on different aspect of the energy deposition process. Both approaches lead to the existence of a maximum of the energy dissipation at a certain depth below the surface, although this aspect disappears in the first approach if a low-order computational method is used. Finally, the crucial point is to model the relative position of this maximum with respect to the maximum penetration depth.

At this point, it seems desirable to devise an empirical model including both the maximum stopping power (Bragg peak) and statistical effects, but this task is outside the scope of this paper.

In the rest of this paper, we concentrate on the numerical approximation of the CSDA approach. We will show that a higher-order approximation dramatically modifies the shape of the SEE yield curve and improves the correlation with experimental results.

\section{NUMERICAL COMPUTATION OF NASCAP's SEE MODEL}

\section{A. Properties of NASCAP's SEE model}

In this section we consider NASCAP's SEE model, which uses the CSDA approach and a double power law for the range [15]. The SEE yield is given by

$$
\delta\left(E_{0}\right)=A \int_{0}^{R\left(E_{0}\right)} S\left(E_{0}, z\right) \exp (-z / \lambda) d z
$$

where

$$
S\left(E_{0}, z\right)=-1 / R^{\prime}(E(z)), \quad R(E)-R\left(E_{0}\right)=z
$$

and $A$ and $\lambda$ are free parameters which will be adjusted to fit experimental data. The range $R(E)$ is given by (5).

We first remark that the SEE yield $\delta$ satisfies the following Ordinary Differential Equation: 


$$
d \delta / d E=A-\delta R^{\prime}(E) / \lambda .
$$

This equation is derived from equation (9) by performing a change of variables $z=z(E)$ in the integral, followed by a differentiation with respect to $E_{0}$.

Applying equation (11) at the energy $E_{\max }$ of the maximum yield where $\mathrm{d} \delta / \mathrm{d} E=0$ gives an interesting relation between $A$ and $\lambda$ :

$$
A=\delta_{\max } R^{\prime}\left(E_{\max }\right) / \lambda .
$$

Then, letting $\mathrm{E} \rightarrow \infty$ in equation (11), we get the high energy asymptotic behavior:

$$
\delta \rightarrow \delta_{\max } R^{\prime}\left(E_{\max }\right) / R^{\prime}(E)=\delta_{\text {max }} S(E) / S\left(E_{\max }\right) .
$$

Note that this is also the $\lambda \rightarrow 0$ limit of $\delta$. As will be seen subsequently, it is crucial for numerical approximations of the SEE yield (3) to be consistent with this asymptotic behavior.

\section{B. Computation of the integral}

Computing an approximation of integral (3) is a difficult task, similar to that of computing special functions. It requires high order approximation methods.

Moreover, as $S\left(E_{0}, z\right)$ is known only implicitly, usual quadrature formulas are not practical. For this reason, low order approximations of $S\left(E_{0}, z\right)$ are often used. The simplest one is to use the following approximation: $S\left(E_{0}, z\right) \approx S\left(E_{0}\right)$ for all $z<R\left(E_{0}\right)$. This gives a first order approximation of $\delta$, similar to the Constant Loss Model (CLM):

$$
\delta(E)=A \lambda \frac{1-\exp (-R(E) / \lambda)}{R^{\prime}(E)}
$$

Such a model is used, e.g. by [6].

The spacecraft charging code NASCAP [15] makes use of $2^{\text {nd }}$ order approximation based on a linearization of $S(z)$ at $z=$ 0 :

$$
S \approx S\left(E_{0}\right)+z \partial_{z} S(z)_{z=0} .
$$

In view of Fig. 2, it is clear that a linear approximation of $S(z)$ does not reproduce the Bragg peak, and therefore underestimates the total stopping power.

The impact on the computed SEE yield will depend on the ratio of the range at maximum stopping power over the escape length, $R\left(E_{\text {Bragg }}\right) / \lambda$. A large ratio corresponds to an SEE dominated by surface phenomena. The maximum of the SEE yield is directly related to the maximum stopping power. If the ratio is small on the other hand, the SEs created relatively deeply inside the material will be able to reach the surface. The energy of the SEE maximum in this case is larger than the energy of the Bragg peak. A high order approximation of the integral (4) is necessary.

Looking at Figure 2 again, it is clear that a higher order approximation is needed to reproduce the shape of the Bragg curve. However, a fourth order expansion at $z=0$ would be cumbersome. Instead, we integrate (11) between $E=0$ and $E$ $=E_{0}$ with a fourth order Backward Differencing Formula with four intermediate steps:

$$
\left\{\begin{aligned}
\delta^{1}=\Delta E /\left(1+R^{\prime}(\Delta E) \Delta E / \lambda\right) \\
\delta^{2}=\left(2 \delta^{1}+\Delta E\right) /\left(1.5+R^{\prime}(2 \Delta E) \Delta E / \lambda\right) \\
\delta^{3}=\left(3 \delta^{2}-1.5 \delta^{1}+\Delta E\right) / \\
\left(11 / 6+R^{\prime}(3 \Delta E) \Delta E / \lambda\right) \\
\delta=A\left(4 \delta^{3}-3 \delta^{2}+4 \delta^{1} / 3+\Delta E\right) / \\
\left(25 / 12+R^{\prime}(4 \Delta E) \Delta E / \lambda\right)
\end{aligned}\right.
$$

where $\Delta \mathrm{E}=\mathrm{E}_{0} / 4$. This method gives a $4^{\text {th }}$ order approximation of the SEE yield $\delta$ which is consistent with the high energy asymptotics (13).

Subsequently, we will call method (16) and the NASCAP approximation (15) the $4^{\text {th }}$ order and the $2^{\text {nd }}$ order methods respectively.

\section{Determination of microscopic parameters: two strategies}

Model (9) involves two free parameters $A$ and $\lambda$. These parameters cannot be measured directly and must be related to SEE yield values. A first strategy consists in using the energy and value of the maximum SEE yield $\delta_{\max }$ and $E_{\max }$. This is the maximum-fitting strategy.

An alternate strategy consists in solving for $\lambda$ such that $\mathrm{d} \delta / \mathrm{d} E\left(E_{\max }\right)=0$, with $A$ given by equation (12). This asymptotic fitting strategy ensures that the correct behavior at infinity is satisfied. On the other hand $\delta\left(E_{\max }\right)=\delta_{\max }$ is not strictly enforced.

In Fig. 3 we have compared the two strategies combined with either a $2^{\text {nd }}$ order or $4^{\text {th }}$ order method, for given values of $\delta_{\max }$ and $E_{\max }$ and a given power-law for the range model. With the maximum-fitting strategy, only the $4^{\text {th }}$ order approximation satisfies the asymptotics of equation (13). A direct consequence is the difference in the predicted second crossover energy. This energy $E_{2}$ defined by $\delta\left(E_{2}\right)=1$ is 1410 $\mathrm{eV}$ for the $4^{\text {th }}$ order method and $1740 \mathrm{eV}$ for the $2^{\text {nd }}$ order method. The relative error is $15 \%$. The same level of error can be expected for computed equilibrium potentials of spacecraft charging simulation.

With the asymptotic-fitting strategy, the actual maximum yield obtained with the $2^{\text {nd }}$ order method is off its real value by $10 \%$. With the $4^{\text {th }}$ order method, the computed maximum is very close to the correct value $\delta_{\max }$ (labeled 'dmax' in Fig. 3). Note that with the asymptotic fitting strategy, the computed values of parameters $A$ and $\lambda$ are similar with the two methods. The difference in the second crossover energy between the two methods is now smaller than $3 \%$.

\section{EXPERIMENTAL DATA}

\section{A. Experimental conditions}

We have analyzed data for three materials, a conductive $\mathrm{Au}$ sample and two insulating samples of Teflon and anodized aluminum. The high purity microcrystalline bulk Au had an estimated RMS surface roughness of less than $10 \mathrm{~nm}$ and surface contamination was less than 0.4 monolayers [17]. A continuous beam method was used to measure the electron yield, with a current density of $\sim 2 \mathrm{nA} / \mathrm{mm}^{2}$ at incident 
energies below $\sim 5 \mathrm{keV}$ and $\sim 50 \mathrm{nA} / \mathrm{mm}^{2}$ above $\sim 5 \mathrm{keV}$ [17]. The $12 \mu \mathrm{m}$ thick FEP Teflon fluoropolymer film with a $\sim 100$ $\mathrm{nm}$ thick vapor deposited Al coating was manufactured by Sheldahl. The anodized aluminum sample was prepared by the Environment Effects Branch at NASA Marshall Space Flight Center. An Al2219 alloy substrate was anodized using a chromic acid etch [18]. The $\mathrm{Al}_{2} \mathrm{O}_{3}$ oxide layer was estimated to be $\sim 1 \mu \mathrm{m}$ thick. The FEP and anodized aluminum samples were studied as received, after only chemical surface cleaning with methanol.

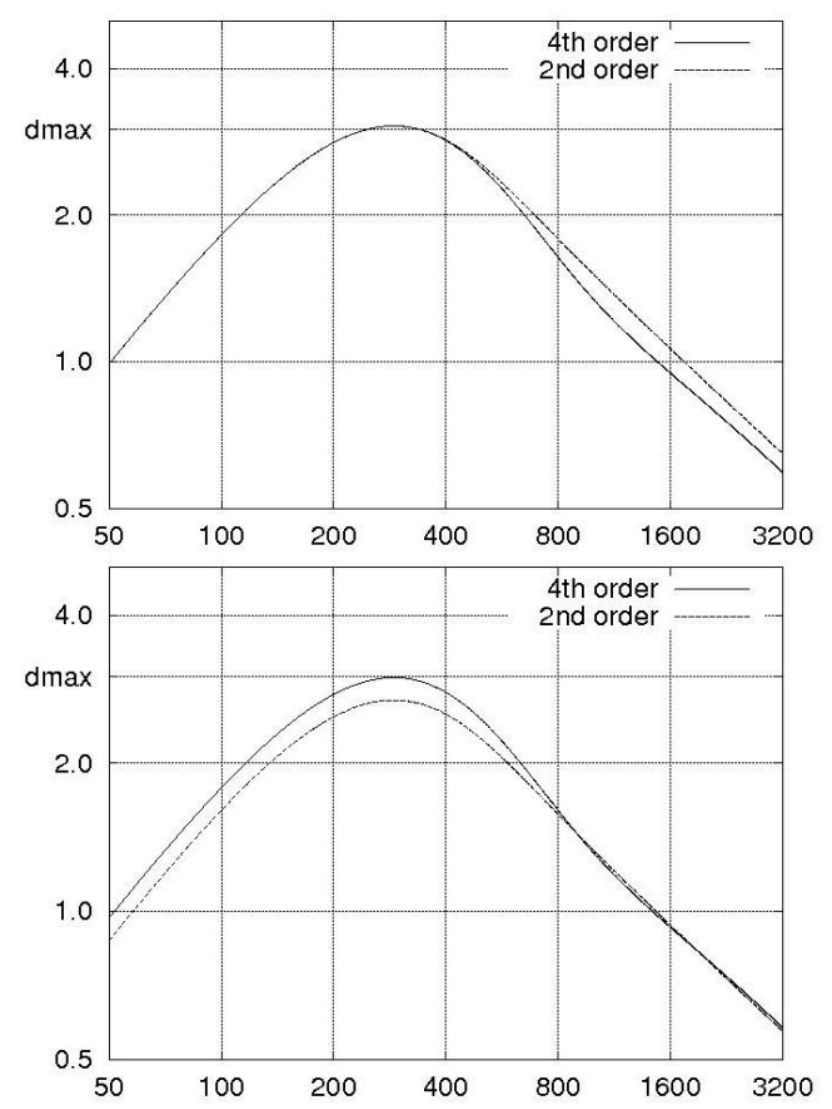

Figure 3: SEE yield versus incident energy (eV). The NASCAP model is computed with a $2^{\text {nd }}$ order (dashed line) or $4^{\text {th }}$ order (full line) method. Top: maximum fitting strategy, bottom: asymptotic fitting.

Data for the Teflon and anodized aluminum samples were acquired using a pulsed beam system, designed to minimize sample charging during the measurements $[5,19]$. Yields were measured with $5 \mu$ s pulses with an $\sim 10 \mathrm{nA}$ beam; between each pulse the sample surface was discharged with a higher flux, low energy electron flood gun and a deuterium UV flux with energy $<6.7 \mathrm{eV}$. Despite this effort to minimize charging, limited charging was observed for these samples, particularly above the second crossover energy.

\section{B. Fitting strategies for experimental data}

For a given material, we can define two characteristic lengths: the range at maximum stopping power $R\left(E_{\text {Bragg }}\right)$ and the escape length of SEs $\lambda$.

If the former is large compared to the latter, then SEE is mostly a surface phenomenon. It is the variation of the stopping power with respect to the incident energy which determines the shape of the SEE yield curve. The Bragg energy corresponds to the energy of the maximum yield. For this type of material, it is useful to use the $\lambda \rightarrow 0$ limit of the NASCAP model, equation (13). Parameter $b_{2}$ is determined by $E_{\text {Bragg }}=E_{\text {max }}$. The SEE yield can be put in the simpler form:

$$
\begin{aligned}
\delta & =\delta_{\max }\left(n_{2}-n_{1}\right) \\
& {\left[\left(n_{2}-1\right)\left(E / E_{\max }\right)^{n_{1}-1}-\left(n_{1}-1\right)\left(E / E_{\max }\right)^{n_{2}-1}\right]^{-1} }
\end{aligned}
$$

We will refer to this model as Model 1 in the figures. It has 4 parameters that can be easily determined:

- $E_{\max }$ and $\delta_{\max }$ from the maximum of SEE yield curve

- $\quad n_{1}-1$ and $n_{2}-1$ are the slopes of the low energy and high energy asymptotes of the SEE yield curve on a log-log plot.

If on the other hand $\lambda$ is larger than $R\left(E_{\text {Bragg }}\right)$, then the SEE yield continues to increase significantly for incident energy $E$ $>E_{\text {Bragg. }}$ In this case, the shape of the SEE yield curve does depend on the value of $\lambda$. In order to reproduce this effect correctly, one has to use a higher order approximation of (3). To simplify the fitting strategy, we use a single power law: $n_{1}$ $=0$. We are thus in the situation where $R\left(E_{\text {bragg }}\right)$ tends to zero. Parameters $E_{\max }$ and $\delta_{\max }$ are determined as before, and $n_{2}$ is adjusted to get the correct high energy asymptote. We will refer to this three-parameter model as Model 2 in the figures.

For completeness, we also study Cazaux' model [13]. Here, parameter $k$ is adjusted to get the correct asymptote at high energy.

\section{Results}

We present numerical results for the three different materials: gold (Fig. 4), Teflon (Fig. 5, top) and anodized aluminum (Fig. 5, bottom). For the first two materials, the fitting strategy based on Model 1 gives a very good fit to experimental data. We were not able to find satisfactory fits with the single power law (Model 2) and the Cazaux model. For the anodized aluminum on the contrary, these two models agree quite well with experimental points, except at low energy. The single power law must however be computed with enough precision. The curve obtained with the $2^{\text {nd }}$ order approximation and the asymptotic fitting (dotted line in Fig. 5) noticeably under-estimates the maximum SEE yield (2.5 predicted instead of 2.7 experimentally). On the other hand, Model 1 is characteristically unable to reproduce the change of concavity of the curve in log-log scale. 

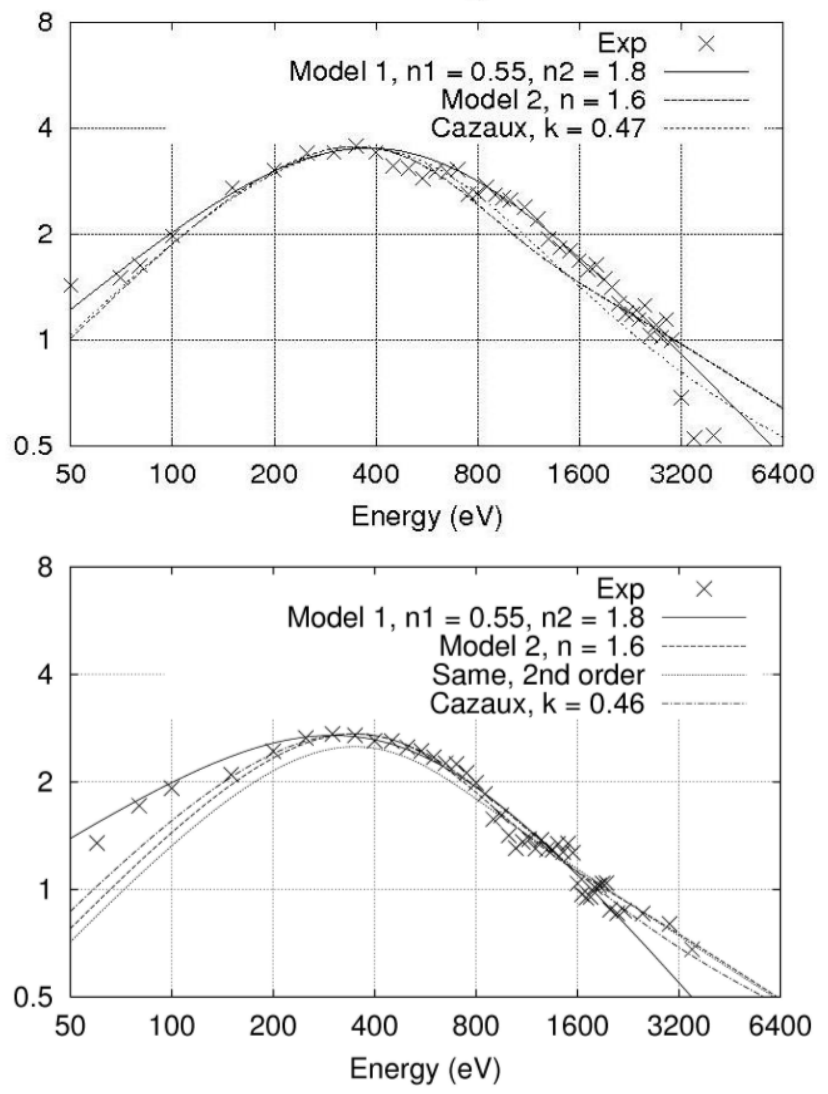

Figure 5: SEE yield for Teflon (top) and anodized aluminum (bottom). Symbols: experimental values, lines: best fit for different models.

\section{CONCLUSION}

In this paper, we have analyzed the properties of a standard model of SEE. We have shown that two cases may occur, depending on the ratio between the range at maximum

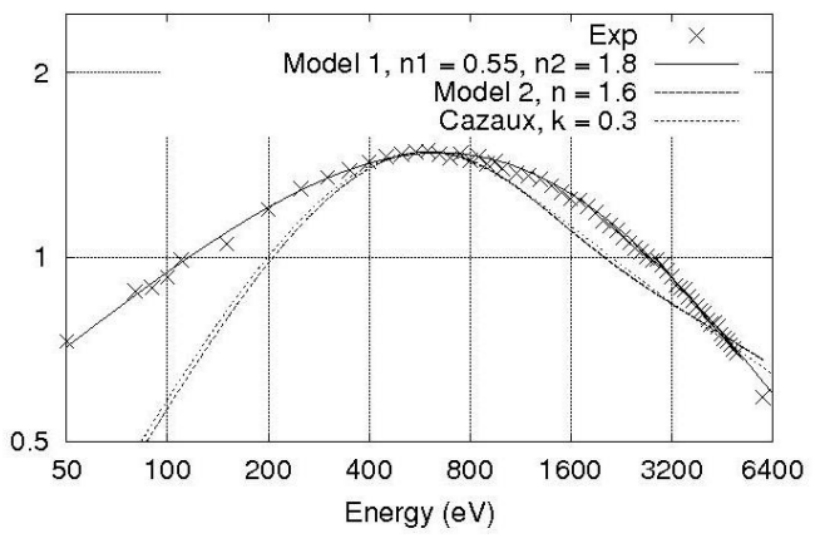

Figure 4: SEE yield curve for gold. Symbols:

experimental points. Dashed lines: best fits for different

stopping power and the escape length.

If this ratio is large, the model can be approximated with a simplified analytic formula. This formula fits experimental data rather well for Gold and Teflon.If the ratio is small, the integral over the whole path length needs to be computed numerically. We have shown that using a low-order method to compute this integral can lead to numerical errors as large as $20 \%$ of the SEE yield. We have also shown that experimental SEE data obtained with an Anodized Aluminum sample are better reproduced by the full SEE model computed with enough accuracy.

In the future, we believe that further modeling and experimental efforts will be needed to analyze the influence of the angle of incidence as well as electric field effects in dielectrics. This is especially important for applications to spacecraft charging where nearly isotropic fluxes and large electric fields are observed.

\section{REFERENCES}

[1] J.G. Laframboise, M. Kamitsuma, R. Godard, "Multiple floating potentials, 'Threshold-temperature' effects and 'Barrier' effects”, in Proc. Int. Symp. On Spacecraft Materials in Space Env., Toulouse, 1982.

[2] I. Katz, M. Mandell, G. Jongeward and M.S. Gussenhoven, "The importance of accurate secondary electron yields in modeling spacecraft charging”, J. Geophysical Research,, 91, 1986, pp. 13739-13744.

[3] J.R. Dennison, C.D. Thomson, J. Kite, V. Zavyalov, J. Corbridge, "Materials Characterization at Utah State University", presented at. $8^{\text {th }}$ Int. Conf. On Solid Dielectrics, Toulouse, 2004.

[4] JR Dennison, C. D. Thomson, and Alec Sim, "The effect of low energy electron and UV/VIS radiation aging on the electron emission properties and breakdown of thin-film dielectrics," in Proceedings of the 8th IEEE Dielectrics and Electrical Insulation Society (DEIS) International Conference on Solid Dielectrics (ICSD), 967-971, (IEEE, Piscataway, NJ, 2004).

[5] J.R. Dennison, Alec Sim, and Clint Thomson, "Evolution of the Electron Yield Curves of Insulators as a Function of Impinging Electron Fluence and Energy," submitted to IEEE Trans. on Plasma Science, 2006.

[6] M.A. Furman, The electron-cloud effect in the arcs of the LHC, CERNLHC-Project-Report-180, 1998.

[7] H. Kimura, I. Mann, "Filtering of the interstellar dust flow near the heliopause: the importance of secondary electron emission for the grain charging”, Earth, Planets \& Space, vol. 51, 1991, pp. 1223-1232.

[8] M.S. Chung and T.E. Everhart, "Simple Calculation of energy distribution of low-energy secondary electrons emitted from metal surfaces under electron bombardment,” J. Applied Physics, 45(2), 707709 (1974).

[9] C.J. Powell and A. Jablonski, NIST Electron Inelastic-Mean-Free-Path Database - Version 1.1, National Institute of Standards and Technology, Gathersburg, MD (2000). Available:http://physics.nist.gov/PhysRefData/Star/Text/ESTAR.html

[10] Reimer, L., Scanning Electron Microscopy. Physics of Image Formation and Microanalysis, (Springer-Verlag, New York, 1985).

[11] Young, J. R., "Penetration of electrons and ions in aluminium," J. Appl. Phys. 27 (1), 1-4 (1956).

[12] S.A. Schwarz, "Application of a semi-empirical sputtering model to SEE”, J. Appl. Phys., 1990.

[13] J. Cazaux, "A new model of dependence: secondary electron emission yield on primary electron energy for application to polymers", J. Phys. D: Appl. Phys., 38, 2005, pp. 2433-2441.

[14] E. M. Baroody, "A Theory of Secondary Electron Emission from Metals", Phys. Rev. 78, 780 (1950).

[15] M.J. Mandell, P.R. Stannard, I. Katz, NASCAP programmer's reference manual, 1984.

[16] JR Dennison, J. Abbott, R. Hoffmann, A. Sim, C.D. Thomson, J. Corbridge, Final Report Part V: Additional Materials Reports, NASA Space Environments and Effects Program Grant, "Electronic Properties of Materials with Application to Spacecraft Charging," 2005. available at http://see.msfc.nasa.gov/ee/db_chargecollector.htm

[17] R. Davies, "An Instrument for Experimental Secondary and Backscattered Electron Investigations with Applications to Space Craft Charging,” MS Thesis, Utah State University, Logan, UT, USA, 1996.

[18] T.A. Schneider, private communication, 2003. 
[19] C. Thomson, "Measurements of the Secondary Electron Emission Properties of Insulators," PhD Dissertation; Utah State University, Logan, UT, USA, 2004. 